\title{
Toe ulceration associated with compression bandaging: observational study
}

\author{
Christopher L H Chan, Felicity J Meyer, Rod J Hay, Kevin G Burnand
}

Foot ulcers can be caused by arterial insufficiency, diabetes mellitus, neuropathy, musculoskeletal disorders, or injury. ${ }^{1}$ The clinical features of an ulcer-such as site, shape, edge, base, and state of the surrounding tissues-often give some indication of its aetiology and aid in further management and treatment. Ulceration of the foot is seen in some patients with venous disease but has not been observed in association with any particular treatment regimen. ${ }^{2}$ We describe a previously unreported finding of ulceration of the toe and cleft, for which there was no demonstrable ischaemic or vasculitic cause, developing in patients treated with compression bandaging for venous ulcers.

\section{Methods and results}

During 1990-2000, 194 new patients were referred to a specialist tertiary centre for the treatment and management of venous leg ulcers. All patients were examined and had a full clinical history taken. Venous leg ulcers were diagnosed according to clinical criteria and confirmed with duplex Doppler ultrasonography, ascending phlebography, and foot volumetry when the ulcers had healed. These patients were managed with standard three or four layer compression treatment. ${ }^{3}$ Bandages were applied from the forefoot to below the knee in a standard manner and changed weekly by fully trained, experienced nurses. Development of any new ulceration during treatment with compression bandaging was investigated by biopsy and microbiological and haematological tests.

Twelve patients (six men, median age 52 (range 32-72) years) who were being treated for characteristic venous leg ulcers around the gaiter region developed foot ulceration after several months of four layer compression bandaging. This ulceration was superficial, with no distinct shape, and was either bilateral (three cases) or unilateral. Ulceration mainly occurred on the dorsum of the medial three toes and interdigital clefts (figure), occasionally extending to the sole of the foot. All of the patients had developed distorted toes after a period of bandaging. Extensive haematological investigations were all normal, as were microbiological culture and viral and fungal antibody titres. Biopsy of the ulcers showed non-specific inflammatory cells with no evidence of malignancy or acid-fast bacilli. No evidence of ischaemia was found-all patients had normal peripheral pulses and Doppler pressures within the normal range (0.9-1.2). No evidence of diabetes mellitus, peripheral neuropathy, underlying osteomyelitis, clinically important oedema, or obesity was found.

These ulcers seemed relatively resistant to healing by conventional methods but improved when the patient rested in bed with the affected foot elevated. They all recurred within a few weeks. Amputation of the toes, although drastic, offers a complete solution and healing. This has been performed successfully in one patient.

\section{Comment}

Toe and cleft ulceration developed de novo in patients treated with four layer compression bandaging for venous ulceration-the ulceration was particularly unusual as it did not share any features typical of ulcers of known aetiology. Fungal infection developing under compression bandages has been noted to cause ulcers of the leg but not of the foot. ${ }^{4}$ No evidence of fungal infection was found in our patients.

The precise mechanism of ulceration is unknown. Increased hypoxia in local tissues, marked oedema of the toes, or development of venous hypertension may be involved. The increased compression pressure of four layer bandages compared with three layer bandages may be a factor. ${ }^{5}$ Alteration of the biomechanics of gait by four layer compression bandaging may also increase friction and compress the toes.

Contributors: CLHC and FJM investigated the patients and were involved in collecting the data and writing the paper. $\mathrm{RJH}$ gave a second opinion on the patients and carried out investigations. KGB first identified the condition and was involved in collecting the data and preparing and writing the paper. CLHC, FJM, and KGB are guarantors for this paper.

Funding: None.

Competing interests: None declared.

Sumpio BE. Foot ulcers. N Engl J Med 2000;343:787-93.

2 Nelzen O, Bergqvist D, Lindhagen A. Venous and non-venous leg ulcers: clinical history and appearance in a population study. Br J Surg 1994;81:182-7.

3 Dale J, Callam M, Ruckley CV. How efficient is a compressive bandage? Nurs Times 1983;79:49-51.

4 Hansson C, Faergemann J, Swanbeck G. Fungal infections occurring under bandages in leg ulcer patients. Acta Derm Venereol 1987;67:341-5.

5 Partsch H, Menzinger G, Blazek V. Static and dynamic measurement of compression pressure. In: Blazek V, Schultz-Ehrenburg U, eds. Frontiers in computer aided visualisation of vascular functions. Berlin: Springer Verlag, 1997:145-52.

(Accepted 10July 2001)

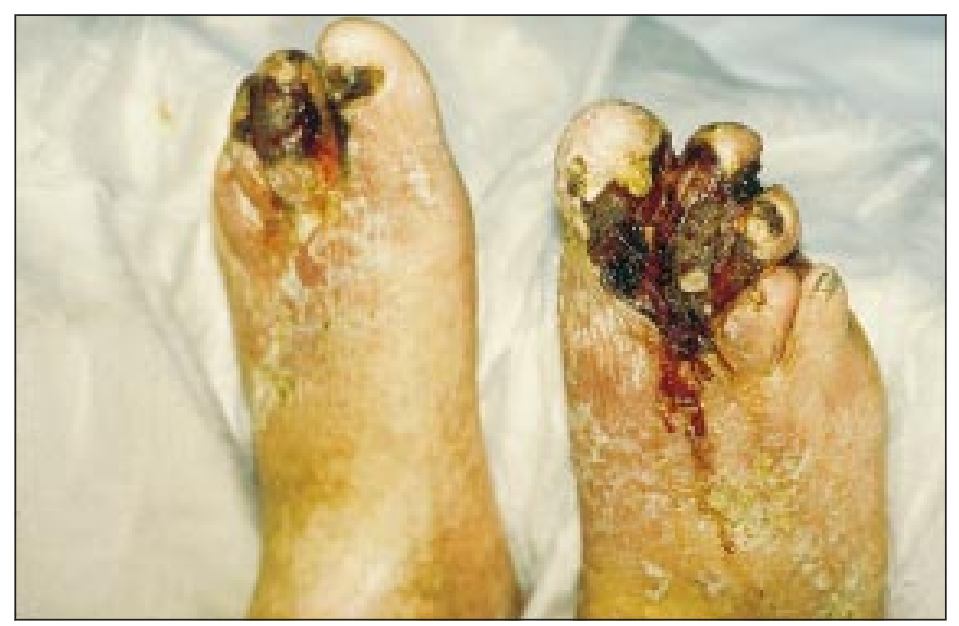

Dorsal view of toe and interdigital cleft ulceration on the dorsum of the first and second toes of the left foot and toe, and interdigital cleft ulceration affecting the first, second, and third toes of the right foot

\section{Department of Surgery, Guy's and St Thomas's NHS Trust, London SE1 7EH \\ Christopher L H Chan specialist registrar Felicity J Meyer specialist registrar Kevin G Burnand professor of surgery St John's Institute of Dermatology, Guy's, King's and St Thomas' School of SE1 7EH Rod J Hay professor of dermatology Correspondence to: K G Burnand kevin.burnand@ kcl.ac.uk}

BMJ 2001;323:1099 